\title{
Some Progress in Conformal Geometry ${ }^{\star}$
}

Sun-Yung A. CHANG ${ }^{\dagger}$, Jie $Q I N G \ddagger$ and Paul YANG ${ }^{\dagger}$

$\dagger$ Department of Mathematics, Princeton University, Princeton, NJ 08540, USA

E-mail: chang@math.princeton.edu,yang@math.princeton.edu

$¥$ Department of Mathematics, University of California, Santa Cruz, Santa Cruz, CA 95064, USA

E-mail: qing@ucsc.edu

Received August 30, 2007, in final form December 07, 2007; Published online December 17, 2007

Original article is available at http://www.emis.de/journals/SIGMA/2007/122/

Abstract. This is a survey paper of our current research on the theory of partial differential equations in conformal geometry. Our intention is to describe some of our current works in a rather brief and expository fashion. We are not giving a comprehensive survey on the subject and references cited here are not intended to be complete. We introduce a bubble tree structure to study the degeneration of a class of Yamabe metrics on Bach flat manifolds satisfying some global conformal bounds on compact manifolds of dimension 4 . As applications, we establish a gap theorem, a finiteness theorem for diffeomorphism type for this class, and diameter bound of the $\sigma_{2}$-metrics in a class of conformal 4-manifolds. For conformally compact Einstein metrics we introduce an eigenfunction compactification. As a consequence we obtain some topological constraints in terms of renormalized volumes.

Key words: Bach flat metrics; bubble tree structure; degeneration of metrics; conformally compact; Einstein; renormalized volume

2000 Mathematics Subject Classification: 53A30; 53C20; 35J60

Dedicated to the memory of Thomas Branson

\section{Conformal gap and finiteness theorem for a class of closed 4-manifolds}

\subsection{Introduction}

Suppose that $\left(M^{4}, g\right)$ is a closed 4-manifold. It follows from the positive mass theorem that, for a 4-manifold with positive Yamabe constant,

$$
\int_{M} \sigma_{2} d v \leq 16 \pi^{2}
$$

and equality holds if and only if $\left(M^{4}, g\right)$ is conformally equivalent to the standard 4-sphere, where

$$
\sigma_{2}[g]=\frac{1}{24} R^{2}-\frac{1}{2}|E|^{2}
$$

$R$ is the scalar curvature of $g$ and $E$ is the traceless Ricci curvature of $g$. This is an interesting fact in conformal geometry because the above integral is a conformal invariant like the Yamabe constant.

\footnotetext{
${ }^{\star}$ This paper is a contribution to the Proceedings of the 2007 Midwest Geometry Conference in honor of Thomas P. Branson. The full collection is available at http://www.emis.de/journals/SIGMA/MGC2007.html
} 
One may ask, whether there is a constant $\epsilon_{0}>0$ such that a closed 4-manifold $M^{4}$ has to be diffeomorphic to $S^{4}$ if it admits a metric $g$ with positive Yamabe constant and

$$
\int_{M} \sigma_{2}[g] d v_{g} \geq(1-\epsilon) 16 \pi^{2}
$$

for some $\epsilon<\epsilon_{0}$ ? Notice that here the Yamabe invariant for such $[g]$ is automatically close to that for the round 4-sphere. There is an analogous gap theorem of Bray and Neves for Yamabe invariant in dimension 3 [4]. One cannot expect the Yamabe invariant alone to isolate the sphere, and it is more plausible to consider the integral of $\sigma_{2}$. We will answer the question affirmatively in the class of Bach flat 4-manifolds.

Recall that Riemann curvature tensor decomposes into

$$
R_{i j k l}=W_{i j k l}+\left(A_{i k} g_{j l}-A_{i l} g_{j k}-A_{j k} g_{i l}+A_{j l} g_{i k}\right),
$$

in dimension 4 , where $W_{i j k l}$ is the Weyl curvature,

$$
A_{i j}=\frac{1}{2}\left(R_{i j}-\frac{1}{6} R g_{i j}\right)
$$

is Weyl-Schouten curvature tensor and $R_{i j}$ is the Ricci curvature tensor. Also recall that the Bach tensor is

$$
B_{i j}=W_{k i j l, l k}+\frac{1}{2} R_{k l} W_{k i j l} .
$$

We say that a metric $g$ is Bach flat if $B_{i j}=0$. Bach flat metrics are critical metrics for the functional $\int_{M}|W|^{2} d v$. Bach flatness is conformally invariant in dimension 4. It follows from Chern-Gauss-Bonnet,

$$
8 \pi^{2} \chi\left(M^{4}\right)=\int_{M}\left(\sigma_{2}+|W|^{2}\right) d v
$$

that $\int_{M} \sigma_{2} d v$ is conformally invariant.

The gap theorem is as follows:

Theorem 1. Suppose that $\left(M^{4},[g]\right)$ is a Bach flat closed 4-manifold with positive Yamabe constant and that

$$
\int_{M}\left(|W|^{2} d v\right)[g] \leq \Lambda_{0}
$$

for some fixed positive number $\Lambda_{0}$. Then there is a positive number $\epsilon_{0}>0$ such that, if

$$
\int_{M} \sigma_{2}[g] d v_{g} \geq(1-\epsilon) 16 \pi^{2}
$$

holds for some constant $\epsilon<\epsilon_{0}$, then $\left(M^{4},[g]\right)$ is conformally equivalent to the standard 4-sphere.

Our approach is based on the recent work on the compactness of Bach flat metrics on 4manifolds of Tian and Viaclovsky [14, 15], and of Anderson [1]. Indeed our work relies on a more precise understanding of the bubbling process near points of curvature concentration. For that purpose we develop the bubble tree structure in a sequence of metrics that describes precisely the concentration of curvature. Our method to develop bubble tree structure is inspired by the work of Anderson and Cheeger [2] on the bubble tree configurations of the degenerations of metrics of bounded Ricci curvature. Our construction is modeled after this work but differs 
in the way that our bubble tree is built from the bubbles at points with the smallest scale of concentration to bubbles with larger scale; while the bubble tree in [2] is constructed from bubbles of large scale to bubbles with smaller scales. The inductive method of construction of our bubble tree is modeled on earlier work of $[3,11,13]$ on the study of concentrations of energies in harmonic maps and the scalar curvature equations.

As a consequence of the bubble tree construction we are able to obtain the following finite diffeomorphism theorem:

Theorem 2. Suppose that $\boldsymbol{A}$ is a collection of Bach flat Riemannian manifolds $\left(M^{4}, g\right)$ with positive Yamabe constant, satisfying

$$
\int_{M}\left(|W|^{2} d v\right)[g] \leq \Lambda_{0}
$$

for some fixed positive number $\Lambda_{0}$, and

$$
\int_{M}\left(\sigma_{2} d v\right)[g] \geq \sigma_{0}
$$

for some fixed positive number $\sigma_{0}$. Then there are only finite many diffeomorphism types in $\boldsymbol{A}$.

It is known that in each conformal class of metrics belonging to the family $\mathbf{A}$, there is a metric $\bar{g}=e^{2 w} g$ such that $\sigma_{2}\left(A_{\bar{g}}\right)=1$, which we shall call the $\sigma_{2}$ metric. The bubble tree structure in the degeneration of Yamabe metrics in $\mathbf{A}$ is also helpful to understand the behavior of the $\sigma_{2}$-metrics in A. For example:

Theorem 3. For the conformal classes $\left[g_{0}\right] \in \boldsymbol{A}$ the conformal metrics $g=e^{2 w} g_{0}$ satisfying the equation $\sigma_{2}(g)=1$ has a uniform bound for the diameter.

The detailed version of this work has appeared in our paper [5].

\subsection{The neck theorem}

The main tool we need to develop the bubble tree picture is the neck theorem which should be compared with the neck theorem in the work of Anderson and Cheeger [2]. Due to the lack of point-wise bounds on Ricci curvature, our version of the neck theorem will have weaker conclusion. But it is sufficient to allow us to construct the bubble tree at each point of curvature concentration.

Let $\left(M^{4}, g\right)$ be a Riemannian manifold. For a point $p \in M$, denote by $B_{r}(p)$ the geodesic ball with radius $r$ centered at $p, S_{r}(p)$ the geodesic sphere of radius $r$ centered at $p$. Consider the geodesic annulus centered at $p$ :

$$
\bar{A}_{r_{1}, r_{2}}(p)=\left\{q \in M: r_{1} \leq \operatorname{dist}(q, p) \leq r_{2}\right\} .
$$

In general, $\bar{A}_{r_{1}, r_{2}}(p)$ may have more than one connected components. We will consider any one component

$$
A_{r_{1}, r_{2}}(p) \subset \bar{A} r_{1}, r_{2}(p)
$$

that meets the geodesic sphere of radius $r_{2}$ :

$$
A_{r_{1}, r_{2}}(p) \bigcap S_{r_{2}}(p) \neq \varnothing \text {. }
$$

Let $H^{3}\left(S_{r}(p)\right)$ be the 3D-Hausdorff measure of the geodesic sphere $S_{r}(p)$. 
Theorem 4. Suppose $\left(M^{4}, g\right)$ is a Bach flat and simply connected 4-manifold with a Yamabe metric of positive Yamabe constant. Let $p \in M, \alpha \in(0,1), \epsilon>0, v_{1}>0$, and $a<\operatorname{dist}(p, \partial M)$. Then there exist positive numbers $\delta_{0}, c_{2}, n$ depending on $\epsilon, \alpha, C_{s}, v_{1}$, a such that the following holds. Let $A_{r_{1}, r_{2}}(p)$ be a connected component of the geodesic annulus in $M$ such that

$$
\begin{aligned}
& r_{2} \leq c_{2} a, \quad r_{1} \leq \delta_{0} r_{2}, \\
& H^{3}\left(S_{r}(p)\right) \leq v_{1} r^{3}, \quad \forall r \in\left[r_{1}, 100 r_{1}\right],
\end{aligned}
$$

and

$$
\int_{A_{r_{1}, r_{2}}(p)}|R m|^{2} d v \leq \delta_{0}
$$

Then $A_{r_{1}, r_{2}}(p)$ is the only such component. In addition, for the only component

$$
A_{\left(\delta_{0}^{-\frac{1}{4}}-\epsilon\right) r_{1},\left(\delta_{0}^{\frac{1}{4}}+\epsilon\right) r_{2}}(p),
$$

which intersects with $S_{\left(\delta_{0}^{\frac{1}{4}}+\epsilon\right) r_{2}}(p)$, there exist some $\Gamma \subset O(4)$, acting freely on $S^{3}$, with $|\Gamma| \leq n$, and an quasi isometry $\psi$, with

$$
A_{\left(\delta_{0}^{-\frac{1}{4}}+\epsilon\right) r_{1},\left(\delta_{0}^{\frac{1}{4}}-\epsilon\right) r_{2}}(p) \subset \Psi\left(C_{\delta_{0}^{-\frac{1}{4}} r_{1}, \delta_{0}^{\frac{1}{4}} r_{2}}\left(S^{3} / \Gamma\right)\right) \subset A_{\left(\delta_{0}^{-\frac{1}{4}}-\epsilon\right) r_{1},\left(\delta_{0}^{\frac{1}{4}}+\epsilon\right) r_{2}}(p)
$$

such that for all $C_{\frac{1}{2} r, r}\left(S^{3} / \Gamma\right) \subset C_{\delta_{0}^{-\frac{1}{4}} r_{1}, \delta_{0}^{\frac{1}{4}} r_{2}}\left(S^{3} / \Gamma\right)$, in the cone coordinates, one has

$$
\left|\left(\Psi^{*}\left(r^{-2} g\right)\right)_{i j}-\delta_{i j}\right|_{C^{1, \alpha}} \leq \epsilon .
$$

The first step in the proof is to use the Sobolev inequality to show the uniqueness of the connected annulus $A_{r_{1}, r_{2}}(p)$. The second step is to establish the growth of volume of geodesic spheres

$$
H^{3}\left(S_{r}(p) \leq C r^{3}\right.
$$

for all $r \in\left[r_{1}, \frac{1}{2} r_{2}\right]$. Here we rely on the work of Tian and Viaclovsky [14, 15] where they analyzed the end structure of a Bach-flat, scalar flat manifolds with finite $L^{2}$ total curvature. The last step is to use the Gromov and Cheeger compactness argument as in the work of Anderson and Cheeger [2] to get the cone structure of the neck.

\subsection{Bubble tree construction}

In this section we attempt to give a clear picture about what happen at curvature concentration points. We will detect and extract bubbles by locating the centers and scales of curvature concentration.

We will assume here that $\left(M_{i}, g_{i}\right)$ are Bach flat 4-manifolds with positive scalar curvature Yamabe metrics, vanishing first homology, and finite $L^{2}$ total curvature. Choose $\delta$ small enough according to the $\epsilon$-estimates and the neck theorem in the previous section. Suppose that $X_{i} \subset M_{i}$ contains a geodesic ball of a fixed radius $r_{0}$ and

$$
\int_{T_{\eta_{0}}\left(\partial X_{i}\right)}\left|R m^{i}\right|^{2} d v^{i} \leq \frac{\delta}{2}
$$

where

$$
T_{\eta_{0}}\left(\partial X_{i}\right)=\left\{p \in M_{i}: \operatorname{dist}\left(p, \partial X_{i}\right)<\eta_{0}\right\},
$$


for some fixed positive number $4 \eta_{0}<r_{0}$. Define, for $p \in X_{i}$,

$$
s_{i}^{1}(p)=r \quad \text { such that } \quad \int_{B_{r}^{i}(p)}\left|R m^{i}\right|^{2} d v^{i}=\frac{\delta}{2} .
$$

Let

$$
p_{i}^{1}=p \quad \text { such that } \quad s_{i}^{1}(p)=\inf _{B_{t_{0}}^{i}\left(p_{i}\right)} s_{i}^{1}(p) .
$$

We may assume $\lambda_{i}^{1}=s_{i}^{1}\left(p_{i}^{1}\right) \rightarrow 0$, for otherwise there would be no curvature concentration in $X_{i}$. We then conclude that $\left(M_{i},\left(\lambda_{i}^{1}\right)^{-2} g_{i}, p_{i}^{1}\right)$ converges to $\left(M_{\infty}^{1}, g_{\infty}^{1}, p_{\infty}^{1}\right)$, which is a Bach flat, scalar flat, complete 4-manifold satisfying the Sobolev inequality, having finite $L^{2}$ total curvature, and one single end.

Definition 1. We call a Bach flat, scalar flat, complete 4-manifold with the Sobolev inequality, finite $L^{2}$ total curvature, and a single ALE end a leaf bubble, while we will call such space with finitely many isolated irreducible orbifold points an intermediate bubble.

Now, we define, for $p \in X_{i} \backslash B_{K^{1} \lambda_{i}^{1}}^{i}\left(p_{i}^{1}\right)$,

$$
s_{i}^{2}(p)=r
$$

such that

$$
\int_{B_{r}^{i}(p) \backslash B_{K^{1} \lambda_{i}^{1}}^{i}\left(p_{i}^{1}\right)}\left|R m^{i}\right|^{2} d v^{i}=\frac{\delta}{2} .
$$

Let

$$
p_{i}^{2}=p
$$

such that

$$
s_{i}^{2}(p)=\inf _{B_{r}^{i}(p) \backslash B_{K^{1} \lambda_{i}^{1}}^{i}\left(p_{i}^{1}\right)} s_{i}^{2}(p) .
$$

Again let $\lambda_{i}^{2}=s_{i}^{2}\left(p_{i}^{2}\right) \rightarrow 0$. Otherwise there would be no more curvature concentration. Then

\section{Lemma 1.}

$$
\frac{\lambda_{i}^{2}}{\lambda_{i}^{1}}+\frac{\operatorname{dist}\left(p_{i}^{1}, p_{i}^{2}\right)}{\lambda_{i}^{1}} \rightarrow \infty .
$$

There are two possibilities:

$$
\begin{array}{ll}
\text { Case 1. } & \frac{\operatorname{dist}\left(p_{i}^{1}, p_{i}^{2}\right)}{\lambda_{i}^{2}} \rightarrow \infty ; \\
\text { Case 2. } & \frac{\operatorname{dist}\left(p_{i}^{1}, p_{i}^{2}\right)}{\lambda_{i}^{2}} \leq M^{1} .
\end{array}
$$

In Case 1, we certainly also have

$$
\frac{\operatorname{dist}\left(p_{i}^{1}, p_{i}^{2}\right)}{\lambda_{i}^{1}} \rightarrow \infty
$$

Therefore, in the convergence of the sequence $\left(M_{i},\left(\lambda_{i}^{2}\right)^{-2} g_{i}, p_{i}^{2}\right)$ the concentration which produces the bubble $\left(M_{\infty}^{1}, g_{\infty}^{1}\right)$ eventually escapes to infinity of $M_{\infty}^{2}$ and hence is not visible to the bubble $\left(M_{\infty}^{2}, g_{\infty}^{2}\right)$, likewise, in the converging sequence $\left(M_{i},\left(\lambda_{i}^{1}\right)^{-2} g_{i}, p_{i}^{1}\right)$ one does not see the concentration which produces $\left(M_{\infty}^{2}, g_{\infty}^{2}\right)$. There are at most finite number of such leaf bubbles. 
Definition 2. We say two bubbles $\left(M_{\infty}^{j_{1}}, g_{\infty}^{j_{1}}\right)$ and $\left(M_{\infty}^{j_{2}}, g_{\infty}^{j_{2}}\right)$ associated with $\left(p_{i}^{j_{1}}, \lambda_{i}^{j_{1}}\right)$ and $\left(p_{i}^{j_{2}}, \lambda_{i}^{j_{2}}\right)$ are separable if

$$
\frac{\operatorname{dist}\left(p_{i}^{j_{1}}, p_{i}^{j_{2}}\right)}{\lambda_{i}^{j_{1}}} \rightarrow \infty \quad \text { and } \quad \frac{\operatorname{dist}\left(p_{i}^{j_{1}}, p_{i}^{j_{2}}\right)}{\lambda_{i}^{j_{2}}} \rightarrow \infty
$$

In Case 2, one starts to trace intermediate bubbles which will be called parents of some bubbles. We would like to emphasize a very important point here. One needs the neck theorem to take limit in Goromov-Hausdorff topology to produce the intermediate bubbles. The neck Theorem is used to prove the limit space has only isolated point singularities, which are then proven to be orbifold points.

Lemma 2. Suppose that there are several separable bubbles $\left\{\left(M_{\infty}^{j}, g_{\infty}^{j}\right)\right\}_{j \in J}$ associated with $\left\{\left(p_{i}^{j}, \lambda_{i}^{j}\right)\right\}_{j \in J}$. Suppose that there is a concentration detected as $\left(p_{i}^{k}, \lambda_{i}^{k}\right)$ after $\left\{\left(p_{i}^{j}, \lambda_{i}^{j}\right)\right\}_{j \in J}$ such that

$$
\frac{\operatorname{dist}\left(p_{i}^{k}, p_{i}^{j}\right)}{\lambda_{i}^{k}} \leq M^{j}
$$

therefore

$$
\frac{\lambda_{i}^{k}}{\lambda_{i}^{j}} \rightarrow \infty
$$

for each $j \in J$. In addition, suppose that $\left\{\left(p_{i}^{j}, \lambda_{i}^{j}\right)\right\}_{j \in J}$ is the maximal collection of such. Then $\left(M_{i},\left(\lambda_{i}^{k}\right)^{-2} g_{i}, p_{i}^{k}\right)$ converges in Gromov-Hausdorff topology to an intermediate bubble $\left(M_{\infty}^{k}, g_{\infty}^{k}\right)$. $\left(M_{\infty}^{k}, g_{\infty}^{k}\right)$ is either a parent or a grandparent of all the given bubbles $\left\{\left(M_{\infty}^{j}, g_{\infty}^{j}\right)\right\}_{j \in J}$.

We remark that it is necessary to create some strange intermediate bubbles to handle the inseparable bubbles. This situation does not arise in the degeneration of Einstein metrics. In that case there is a gap theorem for Ricci flat complete orbifolds and there is no curvature concentration at the smooth points due to a simple volume comparison argument, both of which are not yet available in our current situation. We will call those intermediate bubbles exotic bubbles.

Definition 3. A bubble tree $T$ is defined to be a tree whose vertices are bubbles and whose edges are necks from neck Theorem. At each vertex $\left(M_{\infty}^{j}, g_{\infty}^{j}\right)$, its ALE end is connected, via a neck, to its parent towards the root bubble of $T$, while at finitely many isolated possible orbifold points of $\left(M_{\infty}^{j}, g_{\infty}^{j}\right)$, it is connected, via necks, to its children towards leaf bubbles of $T$. We say two bubble trees $T_{1}$ and $T_{2}$ are separable if their root bubbles are separable.

To finish this process we just iterate the process of extracting bubbles the construction has to end at some finite steps. In summary we have

Theorem 5. Suppose that $\left(M_{i}, g_{i}\right)$ are Bach flat 4-manifolds with positive scalar curvature Yamabe metrics, vanishing first homology, and finite $L^{2}$ total curvature. Then $\left(M_{i}, g_{i}\right)$ converges to Bach-flat 4-manifold $\left(M_{\infty}, g_{\infty}\right)$ with finitely orbifold singularities $S$. The convergence is strong in $C^{\infty}$ away from a finite number of points $B \supset S$. At each point $b$ in $B$ there is a bubble tree attached to $b$. 


\section{Conformally compact Einstein manifolds}

\subsection{Conformally compact Einstein manifolds}

Suppose that $X^{n+1}$ is a smooth manifold of dimension $n+1$ with smooth boundary $\partial X=M^{n}$. A defining function for the boundary $M^{n}$ in $X^{n+1}$ is a smooth function $x$ on $\bar{X}^{n+1}$ such that

$$
\begin{cases}x>0 & \text { in } X \\ x=0 & \text { on } M \\ d x \neq 0 & \text { on } M\end{cases}
$$

A Riemannian metric $g$ on $X^{n+1}$ is conformally compact if $\left(\bar{X}^{n+1}, x^{2} g\right)$ is a compact Riemannian manifold with boundary $M^{n}$ for a defining function $x$. Conformally compact manifold $\left(X^{n+1}, g\right)$ carries a well-defined conformal structure on the boundary $M^{n}$, where each metric $\hat{g}$ in the class is the restriction of $\bar{g}=x^{2} g$ to the boundary $M^{n}$ for a defining function $x$. We call $\left(M^{n},[\hat{g}]\right)$ the conformal infinity of the conformally compact manifold $\left(X^{n+1}, g\right)$. A short computation yields that, given a defining function $x$,

$$
R_{i j k l}[g]=|d x|_{\bar{g}}^{2}\left(g_{i k} g_{j l}-g_{i l} g_{j k}\right)+O\left(x^{3}\right)
$$

in a coordinate $(0, \epsilon) \times M^{n} \subset X^{n+1}$. Therefore, if we assume that $g$ is also asymptotically hyperbolic, then

$$
\left.|d x|_{\bar{g}}^{2}\right|_{M}=1
$$

for any defining function $x$. If $\left(X^{n+1}, g\right)$ is a conformally compact manifold and $\operatorname{Ric}[g]=-n g$, then we call $\left(X^{n+1}, g\right)$ a conformally compact Einstein manifold.

Given a conformally compact, asymptotically hyperbolic manifold $\left(X^{n+1}, g\right)$ and a representative $\hat{g}$ in $[\hat{g}]$ on the conformal infinity $M^{n}$, there is a uniquely determined defining function $x$ such that, on $M \times(0, \epsilon)$ in $X, g$ has the normal form

$$
g=x^{-2}\left(d x^{2}+g_{x}\right)
$$

where $g_{x}$ is a 1-parameter family of metrics on $M$. This is because

Lemma 3. Suppose that $\left(X^{n+1}, g\right)$ is a conformally compact, asymptotically hyperbolic manifold with the conformal infinity $(M,[\hat{g}])$. Then, for any $\hat{g} \in[\hat{g}]$, there exists a unique defining function $x$ such that

$$
|d x|_{r^{2} g}^{2}=1
$$

in a neighborhood of the boundary $[0, \epsilon) \times M$ and

$$
\left.r^{2} g\right|_{M}=\hat{g} .
$$

Given a conformally compact Einstein manifold $\left(X^{n+1}, g\right)$, in the local product coordinates $(0, \epsilon) \times M^{n}$ near the boundary where the metric takes the normal form (1), the Einstein equations split and display some similarity to a second order ordinary differential equations with a regular singular point.

Lemma 4. Suppose that $\left(X^{n+1}, g\right)$ is a conformally compact Einstein manifold with the conformal infinity $\left(M^{n},[\hat{g}]\right)$ and that $x$ is the defining function associated with a metric $\hat{g} \in[\hat{g}]$. Then

$$
g_{x}=\hat{g}+g^{(2)} x^{2}+(\text { even powers of } x)+g^{(n-1)} x^{n-1}+g^{(n)} x^{n}+\cdots,
$$


when $n$ is odd, and

$$
g_{x}=\hat{g}+g^{(2)} x^{2}+(\text { even powers of } x)+g^{(n)} x^{n}+h x^{n} \log x+\cdots,
$$

when $n$ is even, where:

a) $g^{(2 i)}$ are determined by $\hat{g}$ for $2 i<n$;

b) $g^{(n)}$ is traceless when $n$ is odd;

c) the trace part of $g^{(n)}$ is determined by $\hat{g}$ and $h$ is traceless and determined by $\hat{g}$;

d) the traceless part of $g^{(n)}$ is divergence free.

Readers are referred to [9] for more details about the above two lemmas.

\subsection{Examples of conformally compact Einstein manifolds}

Let us look at some examples.

a) The hyperbolic spaces

$$
\left(R^{n+1}, \frac{(d|x|)^{2}}{1+|x|^{2}}+|x|^{2} d \sigma\right)
$$

where $d \sigma$ is the standard metric on the $n$-sphere. We may write

$$
g_{H}=s^{-2}\left(d s^{2}+\left(1-\frac{s^{2}}{4}\right)^{2} d \sigma\right),
$$

where

$$
s=\frac{2}{\sqrt{1+|x|^{2}}+|x|}
$$

is a defining function. Hence the conformal infinity is the standard round sphere $\left(S^{n}, d \sigma\right)$.

b) The hyperbolic manifolds

$$
\left(S^{1}(\lambda) \times R^{n},\left(1+r^{2}\right) d t^{2}+\frac{d r^{2}}{1+r^{2}}+r^{2} d \sigma\right) .
$$

Let

$$
r=\frac{1-\frac{s^{2}}{4}}{s}=\sinh \log \frac{2}{s}
$$

for a defining function $s$. Then

$$
g_{H}^{0}=s^{-2}\left(d s^{2}+\left(1+\frac{s^{2}}{4}\right)^{2} d t^{2}+\left(1-\frac{s^{2}}{4}\right)^{2} d \sigma\right) .
$$

Thus the conformal infinity is standard $\left(S^{1}(\lambda) \times S^{n-1}, d t^{2}+d \sigma\right)$.

c) AdS-Schwarzchild

$$
\left(R^{2} \times S^{2}, g_{+1}^{m}\right),
$$

where

$$
g_{+1}^{m}=V d t^{2}+V^{-1} d r^{2}+r^{2} g_{S^{2}}, \quad V=1+r^{2}-\frac{2 m}{r},
$$


$m$ is any positive number, $r \in\left[r_{h},+\infty\right), t \in S^{1}(\lambda)$ and $(\theta, \phi) \in S^{2}$, and $r_{h}$ is the positive root for $1+r^{2}-\frac{2 m}{r}=0$. In order for the metric to be smooth at each point where $S^{1}$ collapses we need $V d t^{2}+\stackrel{r}{V}^{-1} d r^{2}$ to be smooth at $r=r_{h}$, i.e.

$$
\left.V^{\frac{1}{2}} \frac{d\left(V^{\frac{1}{2}} 2 \pi \lambda\right)}{d r}\right|_{r=r_{h}}=2 \pi .
$$

Note that its conformal infinity is $\left(S^{1}(\lambda) \times S^{2},\left[d t^{2}+d \theta^{2}+\sin ^{\theta} d \phi^{2}\right]\right)$ and $S^{1}$ collapses at the totally geodesic $S^{2}$, which is the so-called horizon. Interestingly, $\lambda$ is does not vary monotonically in $r_{h}$, while $r_{h}$ monotonically depends on $m$. In fact, for each $0<\lambda<1 / \sqrt{3}$, there are two different $m_{1}$ and $m_{2}$ which share the same $\lambda$. Thus, for the same conformal infinity $S^{1}(\lambda) \times S^{2}$ when $0<\lambda<1 / \sqrt{3}$, there are two non-isometric AdS-Schwarzschild space with metric $g_{m_{1}}^{+}$ and $g_{m_{2}}^{+}$on $R^{2} \times S^{2}$. These are the interesting simple examples of non-uniqueness for conformally compact Einstein metrics.

d) AdS-Kerr spaces

$$
\left(C P^{2} \backslash\{p\}, g_{\alpha}\right),
$$

where $p$ is a point on $C P^{2}$,

$$
\begin{aligned}
& g_{\alpha}=E_{\alpha}\left(\left(r^{2}-1\right) F_{\alpha}^{-1} d r^{2}+\left(r^{2}-1\right)^{-1} F_{\alpha}(d t+\cos \theta d \phi)^{2}+\left(r^{2}-1\right)\left(d \theta^{2}+\sin ^{2} \theta d \phi^{2}\right)\right), \\
& E_{\alpha}=\frac{2}{3} \frac{\alpha-2}{\alpha^{2}-1}, \quad F_{\alpha}=(r-\alpha)\left(\left(r^{3}-6 r+3 \alpha^{-1}\right) E_{\alpha}+4\left(r-\alpha^{-1}\right)\right),
\end{aligned}
$$

$r \geq \alpha, t \in S^{1}(\lambda)$, and $(\theta, \phi) \in S^{2}$. For the metric to be smooth at the horizon, the totally geodesic $S^{2}$, we need to require

$$
\sqrt{\frac{F}{E\left(r^{2}-1\right)}} \frac{d}{d r}\left(2 \pi \lambda \sqrt{\frac{E F}{r^{2}-1}}\right)=2 \pi .
$$

Here $(t, \theta, \phi)$ is the coordinates for $S^{3}$ through the Hopf fiberation. The conformal infinity is the Berger sphere with the Hopf fibre of length $\pi E_{\alpha}$ and the $S^{2}$ of area $4 \pi E_{\alpha}$. For every $0<\lambda<(2-\sqrt{3}) / 3$ there are exactly two $\alpha$, hence two AdS-Kerr metrics $g_{\alpha}$. It is interesting to note that $(2-\sqrt{3}) / 3<1$, so the standard $S^{3}(1)$ is not included in this family.

One may ask, given a conformal manifold $\left(M^{n},[\hat{g}]\right)$, is there a conformally compact Einstein manifold $\left(X^{n+1}, g\right)$ such that $\left(M^{n},[\hat{g}]\right)$ is the conformal infinity? This in general is a difficult open problem. Graham and Lee in [10] showed that for any conformal structure that is a perturbation of the round one on the sphere $S^{n}$ there exists a conformally compact Einstein metric on the ball $B^{n+1}$.

\subsection{Conformal compactifications}

Given a conformally compact Einstein manifold $\left(X^{n+1}, g\right)$, what is a good conformal compactification? Let us consider the hyperbolic space. The hyperbolic space $\left(H^{n+1}, g_{H}\right)$ is the hyperboloid

$$
\left\{(t, x) \in R \times R^{n+1}:-t^{2}+|x|^{2}=-1, t>0\right\}
$$

in the Minkowski space-time $R^{1, n+1}$. The stereographic projection via the imaginary south pole gives the Poincaré ball model

$$
\left(B^{n+1},\left(\frac{2}{1-|y|^{2}}\right)^{2}|d y|^{2}\right)
$$


and replacing the $x$-hyperplane by $z$-hyperplane tangent to the light cone gives the half-space model

$$
\left(R_{+}^{n+1}, \frac{|d z|^{2}}{z_{n+1}^{2}}\right)
$$

where

$$
\frac{1+|y|^{2}}{1-|y|^{2}}=t, \quad \frac{1}{z_{n+1}}=t-x_{n+1} .
$$

Therefore

$$
\begin{aligned}
& \left(H^{n+1}, t^{-2} g_{H}\right)=\left(S_{+}^{n+1}, g_{S^{n+1}}\right), \\
& \left(H^{n+1},(t+1)^{-2} g_{H}\right)=\left(B^{n+1},|d y|^{2}\right), \\
& \left(H^{n+1},\left(t-x_{n+1}\right)^{-2} g_{H}\right)=\left(R_{+}^{n+1},|d z|^{2}\right) .
\end{aligned}
$$

The interesting fact here is that all coordinate functions $\left\{t, x_{1}, x_{2}, \ldots, x_{n+1}\right\}$ of the Minkowski space-time are eigenfunctions on the hyperboloid. Thus positive eigenfunctions on a conformally compact Einstein manifold are expected to be candidates for good conformal compactifications. This is first observed in [12].

Lemma 5. Suppose that $\left(X^{n+1}, g\right)$ is a conformally compact Einstein manifold and that $x$ is a special defining function associated with a representative $\hat{g} \in[\hat{g}]$. Then there always exists a unique positive eigenfunction $u$ such that

$$
\Delta u=(n+1) u \quad \text { in } X
$$

and

$$
u=\frac{1}{x}+\frac{R[\hat{g}]}{4 n(n-1)} x+O\left(x^{2}\right)
$$

near the infinity.

We remark here that, for the hyperbolic space $H^{n+1}$ and the standard round metric in the infinity, we have

$$
t=\frac{1}{x}+\frac{1}{4} x
$$

As we expect, positive eigenfunctions indeed give a preferable conformal compactification.

Theorem 6. Suppose that $\left(X^{n+1}, g\right)$ is a conformally compact Einstein manifold, and that $u$ is the eigenfunction obtained for a Yamabe metric $\hat{g}$ of the conformal infinity $(M,[\hat{g}])$ in the previous lemma. Then $\left(X^{n+1}, u^{-2} g\right)$ is a compact manifold with totally geodesic boundary $M$ and

$$
R\left[u^{-2} g\right] \geq \frac{n+1}{n-1} R[\hat{g}]
$$

As a consequence

Corollary 1. Suppose that $\left(X^{n+1}, g\right)$ is a conformally compact Einstein manifold and its conformal infinity is of positive Yamabe constant. Suppose that $u$ is the positive eigenfunction associated with the Yamabe metric on the conformal infinity obtained in Lemma 1. Then $\left(X^{n+1}, u^{-2} g\right)$ is a compact manifold with positive scalar curvature and totally geodesic boundary. 
The work of Schoen-Yau and Gromov-Lawson then give some topological obstruction for a conformally compact Einstein manifold to have its conformal infinity of positive Yamabe constant. A surprising consequence of the eigenfunction compactifications is the rigidity of the hyperbolic space without assuming the spin structure.

Theorem 7. Suppose that $\left(X^{n+1}, g\right)$ is a conformally compact Einstein manifold with the round sphere as its conformal infinity. Then $\left(X^{n+1}, g\right)$ is isometric to the hyperbolic space.

\subsection{Renormalized volume}

We will introduce the renormalized volume, which was first noticed by physicists in their investigations of the holography principles in AdS/CFT. Take a defining function $x$ associated with a choice of the metric $\hat{g} \in[\hat{g}]$ on the conformal infinity, then compute, when $n$ is odd,

$$
\operatorname{Vol}\left(\{x>\epsilon\}=c_{0} \epsilon^{-n}+\text { odd powers of } \epsilon+V+o(1)\right.
$$

when $n$ is even,

$$
\operatorname{Vol}(\{s>\epsilon\})=c_{0} \epsilon^{-n}+\text { even powers of } \epsilon+L \log \frac{1}{\epsilon}+V+o(1) .
$$

It turns out the numbers $V$ in odd dimension and $L$ in even dimension are independent of the choice of the metrics in the class. We will see that $V$ in even dimension is in fact a conformal anomaly.

Lemma 6. Suppose that $\left(X^{n+1}, g\right)$ is a conformally compact Einstein manifold and that $\bar{x}$ and $x$ are two defining functions associated with two representatives in $[\hat{g}]$ on the conformal infinity $\left(M^{n},[\hat{g}]\right)$. Then

$$
\bar{x}=x e^{w}
$$

for a function $w$ on a neighborhood of the boundary $[0, \epsilon) \times M$ whose expansion at $x=0$ consists of only even powers of $x$ up through and including $x^{n+1}$ term.

Theorem 8. Suppose that $\left(X^{n+1}, g\right)$ is a conformally compact Einstein manifold. The $V$ in (2) when $n$ is odd and $L$ in (3) when $n n$ is even are independent of the choice of representative $\hat{g} \in[\hat{g}]$ on the conformal infinity $\left(M^{n},[\hat{g}]\right)$.

Let us calculate the renormalized volume for the examples in Section 2.2.

a) The hyperbolic space: We recall

$$
\left(H^{4}, g_{H}\right)=\left(B^{4},\left(\frac{2}{1-|y|^{2}}\right)^{2}|d y|^{2}\right),
$$

where

$$
g_{H}=s^{-2}\left(d s^{2}+\left(1-\frac{s^{2}}{4}\right)^{2} h_{0}\right)
$$

and $h_{0}$ is the round metric on $S^{3}$. Then

$$
\operatorname{vol}(\{s>\epsilon\})=\int_{\epsilon}^{2} \int_{S^{3}} s^{-4}\left(1-\frac{s^{2}}{4}\right)^{3} d \sigma_{0} d s
$$


where $d \sigma_{0}$ is the volume element for the round unit sphere

$$
\begin{aligned}
\operatorname{vol}(\{s>\epsilon\}) & =2 \pi^{2} \int_{\epsilon}^{2} s^{-4}\left(1-\frac{3 s^{2}}{4}+\frac{3 s^{4}}{16}-\frac{s^{6}}{64}\right) d s \\
& =2 \pi^{2}\left(-\left.\frac{1}{3} s^{-3}\right|_{\epsilon} ^{2}+\left.\frac{3}{4} s^{-1}\right|_{\epsilon} ^{2}+\frac{3}{16}(2-\epsilon)-\left.\frac{1}{3 \times 64} s^{3}\right|_{\epsilon} ^{2}\right) \\
& =\frac{2 \pi^{2}}{3} \epsilon^{-3}-\frac{3 \pi^{2}}{2} \epsilon^{-1}+2 \pi^{2}\left(-\frac{1}{3 \times 8}+\frac{3}{8}+\frac{3}{8}-\frac{1}{3 \times 8}\right)+O(\epsilon) \\
& =\frac{2 \pi^{2}}{3} \epsilon^{-3}-\frac{3 \pi^{2}}{2} \epsilon^{-1}+\frac{4 \pi^{2}}{3}+O(\epsilon) .
\end{aligned}
$$

Thus

$$
V\left(H^{4}, g_{H}\right)=\frac{4 \pi^{2}}{3} .
$$

b) The hyperbolic manifold: We recall

$$
\left(S^{1}(\lambda) \times R^{3},\left(1+r^{2}\right) d t^{2}+\frac{d r^{2}}{1+r^{2}}+r^{2} g_{S^{2}}\right)
$$

and

$$
g_{H}^{0}=s^{-2}\left(d s^{2}+\left(1-\frac{s^{2}}{4}\right)^{2}\left(d \theta^{2}+\sin ^{2} \theta d \phi^{2}\right)+\left(1+\frac{s^{2}}{4}\right)^{2} d t^{2}\right) .
$$

Then

$$
\operatorname{vol}(\{s>\epsilon\})=\int_{\epsilon}^{2} \int_{S^{2}} \int_{S^{1}} s^{-4}\left(1-\frac{s^{2}}{4}\right)^{2}\left(1+\frac{s^{2}}{4}\right) d \omega_{0} d t d s
$$

where $d \omega_{0}$ stands for the volume element for the round unit sphere $S^{2}$

$$
\begin{aligned}
\operatorname{vol}(\{s>\epsilon\}) & =8 \pi^{2} \int_{\epsilon}^{2} s^{-4}\left(1-\frac{s^{2}}{2}+\frac{s^{4}}{16}\right)\left(1+\frac{s^{2}}{4}\right) d s \\
& =8 \pi^{2} \lambda \int_{\epsilon}^{2} s^{-4}\left(1-\frac{s^{2}}{4}-\frac{s^{4}}{16}+\frac{s^{6}}{64}\right) d s \\
& =\frac{8 \pi^{2}}{3} \lambda \epsilon^{-3}-2 \pi^{2} \lambda \epsilon^{-1}+8 \pi^{2} \lambda\left(-\frac{1}{3 \times 8}+\frac{1}{8}-\frac{1}{8}+\frac{1}{3 \times 8}\right)+O(\epsilon) \\
& =\frac{8 \pi^{2}}{3} \lambda \epsilon^{-3}-2 \pi^{2} \lambda \epsilon^{-1}+O(\epsilon) .
\end{aligned}
$$

Thus

$$
V\left(S^{1} \times R^{3}, g_{H}^{0}\right)=0 .
$$

c) AdS-Schwarzschild spaces: We recall on $S^{2} \times R^{2}$

$$
g_{+1}^{m}=\left(1+r^{2}-\frac{2 m}{r}\right) d t^{2}+\frac{d r^{2}}{1+r^{2}-\frac{2 m}{r}}+r^{2}\left(d \theta^{2}+\sin ^{2} \theta d \phi^{2}\right) .
$$

First let us find the special defining function, i.e. to have

$$
\frac{1}{1+r^{2}-\frac{2 m}{r}} d r^{2}=s^{-2} d s^{2}
$$


that is, if denote by $r=\rho / s$, where $\rho=\rho(s)$,

$$
\rho-s \rho^{\prime}=\sqrt{\rho^{2}+s^{2}-2 m s^{3} / \rho},
$$

and $\rho(0)=1$. One may solve it in power series

$$
\rho=1-\frac{1}{4} s^{2}+\frac{m}{3} s^{3}+\cdots .
$$

Then

$$
g_{+1}^{m}=s^{-2}\left(d s^{2}+\left(\rho^{2}+s^{2}-\frac{2 m s^{3}}{\rho}\right) d t^{2}+\rho^{2}\left(d \theta^{2}+\sin ^{2} \theta d \phi^{2}\right)\right) .
$$

Note that $s \in\left[\epsilon, s_{h}\right]$ for $r \in\left[r_{h}, M_{\epsilon}\right]$,

$$
\log s_{h}=\log \epsilon+\int_{r_{h}}^{M_{\epsilon}} \frac{1}{\sqrt{1+r^{2}-\frac{2 m}{r}}} d r<+\infty,
$$

and

$$
M_{\epsilon}=\epsilon^{-1} \rho(\epsilon)=\epsilon^{-1}\left(1-\frac{1}{4} \epsilon^{2}+\frac{m}{3} s^{3}+\cdots\right) .
$$

Therefore

$$
\begin{aligned}
\operatorname{vol}(\{s>\epsilon\}) & =\int_{\epsilon}^{s_{h}} \int_{S^{1}(\lambda)} \int_{S^{2}} s^{-4} \sqrt{\rho^{2}+s^{2}-\frac{2 m s^{2}}{\rho}} \rho^{2} d t d \sigma_{0} d s \\
& =8 \pi^{2} \lambda \int_{\epsilon}^{s_{h}} s^{-4} \sqrt{\rho^{2}+s^{2}-\frac{2 m s^{3}}{\rho}} \rho^{2} d s \\
& =8 \pi^{2} \lambda \int_{r_{h}}^{M} s^{-1} \sqrt{1+r^{2}-\frac{2 m}{r}} r^{2}\left(-\frac{d s}{d r}\right) d r \\
& =8 \pi^{2} \lambda \int_{r_{h}}^{M} r^{2} d r=\frac{8 \pi^{2} \lambda}{3}\left(M^{3}-r_{h}^{3}\right) .
\end{aligned}
$$

Thus the renormalized volume

$$
V\left(R^{2} \times S^{2}, g_{+1}^{m}\right)=\frac{8 \pi^{2}}{3} \frac{r_{h}^{2}\left(1-r_{h}^{2}\right)}{3 r_{h}^{2}+1},
$$

where $V\left(R^{2} \times S^{2}, g_{+1}^{m}\right)<0$ when $r_{h}>1 ; V\left(R^{2} \times S^{2}, g_{+1}^{m}\right)=0$ only when $r_{h}=1$ or 0 ; and it achieves its maximum value at $r_{h}=1 / \sqrt{3}$

$$
V\left(R^{2} \times S^{2}, g_{+1}^{m}\right)_{\max }=\frac{1}{9} \cdot \frac{4 \pi^{2}}{3} \chi\left(R^{2} \times S^{2}\right) .
$$

d) AdS-Kerr spaces: We will omit the calculation here. The renormalized volume

$$
V\left(\mathrm{CP}^{2} \backslash\{p\}, g_{\alpha}\right)=4 \pi^{2} E_{\alpha}\left(-\frac{1}{6} E_{\alpha}\left(\alpha^{3}+3 \alpha^{-1}\right)+\frac{2}{3}\left(\alpha+\alpha^{-1}\right)\right) .
$$

Clearly, $V\left(\mathrm{CP}^{2} \backslash\{p\}, g_{\alpha}\right)$ goes to zero when $\alpha$ goes to 2 , and $V\left(\mathrm{CP}^{2} \backslash\{p\}, g_{\alpha}\right)$ goes to $-\infty$ when $\alpha$ goes to $\infty$. One may find the maximum value for the renormalized volume is achieved at $\alpha=2+\sqrt{3}$. Therefore

$$
V\left(\mathrm{CP}^{2} \backslash\{p\}, g_{\alpha}\right)_{\max }=\frac{4 \pi^{2}}{3} \cdot \frac{2(4-\sqrt{3})}{9}<\frac{1}{2} \cdot \frac{4 \pi^{2}}{3} \chi\left(\mathrm{CP}^{2} \backslash\{p\}\right) .
$$




\subsection{Renormalized volume and Chern-Gauss-Bonnet formula}

We start with the Gauss-Bonnet formula on a surface $\left(M^{2}, g\right)$

$$
4 \pi \chi\left(M^{2}\right)=\int_{M} K d v_{g}
$$

where $K$ is the Gaussian curvature of $\left(M^{2}, g\right)$. The transformation of the Gaussian curvature under a conformal change of metrics $g_{w}=e^{2 w} g$ is governed by the Laplacian as follows:

$$
-\Delta_{g} w+K[g]=K\left[e^{2 w} g\right] e^{2 w} .
$$

The Gauss-Bonnet formula for a compact surface with boundary $\left(M^{2} g\right)$ is

$$
4 \pi \chi(M)=\int_{M} K d V_{g}+2 \int_{\partial M} k d \sigma_{g}
$$

where $k$ is the geodesic curvature for $\partial M$ in $(M, g)$. The transformation of the geodesic curvature under a conformal change of metric $g_{w}=e^{2 w} g$ is

$$
-\partial_{n} w+k[g]=k\left[e^{2 w} g\right] e^{w}
$$

where $\partial_{n}$ is the inward normal derivative. Notice that

$$
-\Delta\left[e^{2 w} g\right]=e^{-2 w}(-\Delta[g]), \quad-\partial_{n}\left[e^{2 w} g\right]=e^{-w}\left(-\partial_{n}[g]\right),
$$

for which we say they are conformally covariant. In four dimension there is a rather complete analogue. We may write the Chern-Gauss-Bonnet formula in the form

$$
8 \pi^{2} \chi\left(M^{4}\right)=\int_{M}\left(|W|^{2}+Q\right) d V_{g}
$$

for closed 4-manifold and

$$
8 \pi^{2} \chi\left(M^{4}\right)=\int_{M}\left(|W|^{2}+Q\right) d V_{g}+2 \int_{\partial M}(L+T) d \sigma_{g}
$$

where $W$ is the Weyl curvature, $L$ is a point-wise conformal invariant curvature of $\partial M$ in $(M, g)$.

$$
\begin{aligned}
Q & =\frac{1}{6}\left(R^{2}-3|\mathrm{Ric}|^{2}-\Delta R\right), \\
T & =-\frac{1}{12} \partial n R+\frac{1}{6} R H-R_{\alpha n \beta n} L_{\alpha \beta}+\frac{1}{9} H^{3}-\frac{1}{3} \operatorname{Tr} L^{3}-\frac{1}{3} \tilde{\Delta} H
\end{aligned}
$$

$R$ is the scalar curvature, Ric is the Ricci curvature, $L$ is the second fundamental form of $\partial M$ in $(M, g)$. We know the transformation of $Q$ under a conformal change metric $g_{w}=e^{2 w} g$ is

$$
P_{4}[g] w+Q[g]=Q\left[e^{2 w} g\right] e^{4 w},
$$

where

$$
P_{4}=(-\Delta)^{2}+\delta\left\{\frac{2}{3} R g-2 \mathrm{Ric}\right\} d
$$

is the so-called Paneitz operator, and the transformation of $T$ is

$$
P_{3}[g] w+T[g]=T\left[e^{2 w}\right] e^{3 w},
$$


where

$$
P_{3}=\frac{1}{2} \partial_{n} \Delta_{g}-\tilde{\Delta} \partial_{n}+\frac{2}{3} H \tilde{\Delta}+L_{\alpha \beta} \tilde{\nabla}_{\alpha} \tilde{\nabla}_{\beta}+\frac{1}{3} \tilde{\nabla}_{\alpha} H \cdot \tilde{\nabla}_{\alpha}+\left(F-\frac{1}{3} R\right) \partial_{n} .
$$

We also have

$$
P_{4}\left[e^{2 w} g\right]=e^{-4 w} P_{4}[g], \quad P_{3}\left[e^{2 w} g\right]=e^{-3 w} P_{3}[g] .
$$

On the other hand, to calculate the renormalized volume in general, for odd $n$, upon a choice of a special defining function $x$, one may solve

$$
-\Delta v=n \quad \text { in } X^{n+1}
$$

for

$$
v=\log x+A+B x^{n},
$$

$A, B$ are even in $x$, and $\left.A\right|_{x=0}=0$. Let

$$
B_{n}[g, \hat{g}]=\left.B\right|_{x=0} .
$$

Fefferman and Graham observed

\section{Lemma 7.}

$$
V\left(X^{n+1}, g\right)=\int_{M} B_{n}[g, \hat{g}] d v[\hat{g}] .
$$

We observe that the function $v$ in the above is also good in conformal compactifications. For example, given a conformally compact Einstein 4-manifold $\left(X^{4}, g\right)$, let us consider the compactification $\left(X^{4}, e^{2 v} g\right)$. Then

$$
Q_{4}\left[e^{2 v} g\right]=0
$$

and its boundary is totally geodesic in $\left(X^{4}, e^{2 v} g\right)$. Moreover

$$
T\left[e^{2 v} g\right]=3 B_{3}[g, \hat{g}] .
$$

Therefore we obtain easily the following generalized Chern-Gauss-Bonnet formula.

Proposition 1. Suppose that $\left(X^{4}, g\right)$ is a conformally compact Einstein manifold. Then

$$
8 \pi^{2} \chi\left(X^{4}\right)=\int_{X^{4}}\left(|W|^{2} d v\right)[g]+6 V\left(X^{4}, g\right) .
$$

\subsection{Topology of conformally compact Einstein 4-manifolds}

In the following let us summarize some of our works appeared in [6]. From the generalized Chern-Gauss-Bonnet formula, obviously

$$
V \leq \frac{4 \pi^{2}}{3} \chi(X)
$$

and the equality holds if and only if $\left(X^{4}, g\right)$ is hyperbolic. Comparing with Chern-Gauss-Bonnet formula for a closed 4-manifold

$$
\frac{1}{8 \pi^{2}} \int_{M^{4}}\left(|W|^{2}+\sigma_{2}\right) d v=\chi\left(M^{4}\right)
$$


one sees that the renormalized volume replaces the role of the integral of $\sigma_{2}$. In the following we will report some results on the topology of a conformally compact Einstein 4-manifold in terms of the size of the renormalized volume relative to the Euler number, which is analogous to the results of Chang-Gursky-Yang [7,8] on a closed 4-manifold with positive scalar curvature and large integral of $\sigma_{2}$ relative to the Euler number. The proofs mainly rely on the conformal compactifications discussed earlier, a simple doubling argument and applications of the above mentioned results of Chang-Gursky-Yang [7, 8].

Theorem 9. Suppose $\left(X^{4}, g\right)$ is a conformally compact Einstein 4-manifold with its conformal infinity of positive Yamabe constant and the renormalized volume $V$ is positive. Then $H^{1}(X, R)=0$.

Theorem 10. Suppose $\left(X^{4}, g\right)$ is a conformally compact Einstein 4-manifold with conformal infinity of positive Yamabe constant. Then

$$
V>\frac{1}{3} \frac{4 \pi^{2}}{3} \chi(X)
$$

implies that $H^{2}(X, R)$ vanishes.

A nice way to illustrate the above argument is the following. We may consider the modified Yamabe constant

$$
Y^{\lambda}(M,[g])=\inf _{g \in[g]} \frac{\int_{M}\left(R[g]+\lambda\left|W^{+}\right|_{g}\right) d v_{g}}{\left(\int_{M} d v_{g}\right)^{\frac{n-2}{n}}} .
$$

Then, one knows that $(M,[g])$ is of positive $Y^{\lambda}(M,[g])$ if and only if there is a metric $g \in[g]$ with $R+\lambda\left|W^{+}\right|>0$. As a consequence of the following Bochner formula

$$
\Delta \frac{1}{2}|\omega|^{2}=|\nabla \omega|^{2}-2 W^{+}(\omega, \omega)+\frac{1}{3} R|\omega|^{2} \geq|\nabla \omega|^{2}+\left(R-2 \sqrt{6}\left|W^{+}\right|\right)|\omega|^{2}
$$

for any self-dual harmonic 2-form $\omega$, one easily sees that a closed oriented 4-manifold with $Y^{-2 \sqrt{6}}>0$ has its $b_{2}^{+}=0$. We also observe

Theorem 11. Suppose $\left(X^{4}, g\right)$ is a conformally compact Einstein 4-manifold with its conformal infinity of positive Yamabe constant and that

$$
V>\frac{1}{2} \frac{4 \pi^{2}}{3} \chi(X)
$$

Then $X$ is diffeomorphic to $B^{4}$ and more interestingly $M$ is diffeomorphic to $S^{3}$.

The detailed proofs of the above theorems are in our paper [6]. One may recall
a) $V\left(H^{4}, g_{H}\right)=\frac{4 \pi^{2}}{3}$
b) $V\left(S^{1} \times R^{3}, g_{H}\right)=0$,
c) $V\left(S^{2} \times R^{2}, g_{+}^{m}\right)=\frac{8 \pi^{2}}{3} \frac{r_{h}^{2}\left(1-r_{h}^{2}\right)}{3 r_{h}^{2}+1} \leq \frac{1}{9} \frac{4 \pi^{2}}{3} \chi\left(S^{2} \times R^{2}\right)$,
d) $V\left(C P^{2} \backslash B, g_{K}\right) \leq \frac{4 \pi^{2}}{3} \frac{2(4-\sqrt{3})}{9}<\frac{1}{3} \frac{4 \pi^{2}}{3} \chi\left(C P^{2} \backslash B\right)$.

Theorem 10 is rather sharp, in cases (c) and (d) the second homology is nontrivial while the renormalized volume is very close to one-third of the maximum. 


\section{References}

[1] Anderson M., Orbifold compactness for spaces of Riemannian metrics and applications, Math. Ann. 331 (2005), 739-778, math.DG/0312111.

[2] Anderson M., Cheeger J., Diffeomorphism finiteness for manifolds with Ricci curvature and $L^{n / 2}$-norm of curvature bounded, Geom. Funct. Anal. 1 (1991), 231-252.

[3] Brezis H., Coron J.M., Convergence of solutions of H-systems or how to blow bubbles, Arch. Ration. Mech. Anal. 89 (1985), 21-56.

[4] Bray H., Neves A., Classification of prime 3-manifolds with Yamabe invariant larger than $R P^{3}$, Ann. of Math. (2) 159 (2004), 407-424.

[5] Chang S.-Y.A., Qing J., Yang P., On a conformal gap and finiteness theorem for a class of four-manifolds. Geom. Funct. Anal. 17 (2007), 404-434, math.DG/0508621.

[6] Chang S.-Y.A., Qing J., Yang P., On the topology of conformally compact Einstein 4-manifolds, in Noncompact Problems at the Intersection of Geometry, Analysis, and Topology, Contemp. Math. 350 (2004), 49-61, math.DG/0305085.

[7] Chang S.-Y.A., Gursky M., Yang P., An equation of Monge-Ampére type in conformal geometry and 4manifolds of positive Ricci curvature, Ann. of Math. (2) 155 (2002), 709-787, math.DG/0409583.

[8] Chang S.-Y.A., Gursky M., Yang P., An apriori estimate for a fully nonlinear equation on 4-manifolds, J. D'Analyse Math. 87 (2002), 151-186.

[9] Graham C.R., Volume and area renormalizations for conformally compact Einstein metrics, in The Proceedings of the 19th Winter School "Geometry and Physics" (1999, Srnì), Rend. Circ. Mat. Palermo (2) 63 (2000), suppl., 31-42, math.DG/9909042.

[10] Graham C.R., Lee J., Einstein metrics with prescribed conformal infinity on the ball, Adv. Math. 87 (1991), 186-225.

[11] Qing J., On singularities of the heat flow for harmonic maps from surfaces into spheres, Comm. Anal. Geom. 3 (1995), 297-315.

[12] Qing J., On the rigidity for conformally compact Einstein manifolds, Int. Math. Res. Not. 2003 (2003), 1141-1153, math.DG/0305084.

[13] Struwe M., Global compactness result for elliptic boundary value problem involving limiting nonlinearities, Math. Z. 187 (1984), 511-517.

[14] Tian G., Viaclovsky J., Bach flat asymptotically ALE metrics, Invent. Math. 160 (2005), 357-415, math.DG/0310302.

[15] Tian G., Viaclovsky J., Moduli space of critical Riemannian metrics in dimension 4, Adv. Math. 196 (2005), 346-372, math.DG/0312318. 